\title{
On the Trivariate Non-Central Chi-Squared Distribution
}

\author{
K. D. P. Dharmawansa*, R. M. A. P. Rajatheva* and C. Tellambura ${ }^{\dagger}$ \\ *Telecommunications Field of Study, Asian Institute of Technology \\ P.O.Box 4, Klong Luang 12120, Pathumthani, Thailand, Email:rajath@ait.ac.th \\ ${ }^{\dagger}$ Department of Electrical and Computer Engineering, University of Alberta, \\ Edmonton, AB T6G 2V4, Canada (Email: chintha@ece.ualberta.ca)
}

\begin{abstract}
In this paper, we derive a new infinite series representation for the trivariate Non-central chi-squared distribution when the underlying correlated Gaussian variables have tridiagonal form of inverse covariance matrix. We make use of the Miller's approach and the Dougall's identity to derive the joint density function. Moreover, the trivariate cumulative distribution function (cdf) and characteristic function (chf) are also derived. Finally, bivariate noncentral chi-squared distribution and some known forms are shown to be special cases of the more general distribution. However, noncentral chi-squared distribution for an arbitrary covariance matrix seems intractable with the Miller's approach.
\end{abstract}

\section{INTRODUCTION}

The $\chi^{2}$ and non-central $\chi^{2}$ distributions play a major role in the performance analysis of communication systems [1]-[5]. The generalized chi-squared distribution is analyzed in detail in [6]-[9]. Khaled and Williams [10] derive a relationship between non-central $\chi^{2}$ distribution and the distribution of generalized Hermite quadratic form. It is well known that the diagonal elements of a Wishart matrix has chi-squared distribution [11]. Joint density of the diagonal elements of a real central Wishart matrix (i.e., multivariate central $\chi^{2}$ distribution) is analyzed in [12]-[14]. The multivariate generalized Rayleigh density studied in [15] is also another form of multivariate central $\chi^{2}$ distribution. Nevertheless, the authors in [15], [16] assumed a tridiagonal form of inverse correlation matrix for the underlying Gaussian variables to derive a closed form solution for the generalized Rayleigh density. However, the trivariate generalized Rayleigh density for an arbitrary correlation matrix is given in [16]. Recently Hagedorn et al [11] derive a trivariate central chisquare distribution from the diagonal elements of a complex Wishart matrix. Miller's assumption of tridiagonal form of inverse correlation matrix is significant since it gives rise to a closed form solution for the multivariate Rayleigh density. Karagiannadis et al [17], [18] have extended the Millers result to $n$ variate Nakagami- $m$ distribution, which is also a some form of multivariate central $\chi^{2}$ density.

It is obvious that the Rice density is closely coupled with the non-central $\chi^{2}$ distribution [19]. The multivariate noncentral $\chi^{2}$ distribution can be thought of as a generalization of the multivariate Rician distribution. The bivariate Rician density is given in [20], [21]. In [22] propose an infinite series representation involving modified Bessel functions of the first kind for the distribution of trivariate Rician distribution when the underlying Gaussian components have a tridiagonal form of inverse covariance matrix. Miller [16] propose an infinite series representation involving modified Bessel function of the first kind for the distribution of bivariate generalized Rician distribution. A careful study of the previous work related to the multivariate distributions reveals that there exists no joint distribution for trivariate noncentral $\chi^{2}$ distribution.

Having motivated with that reason we propose a novel expressions for the trivariate noncentral chi-squared distribution, cdf and chf when the underlying Gaussian components have a tridiagonal form of inverse covariance matrix. Our main derivation is inspired with the approach due to Miller [16] and a theorem for a product of ultraspherical polynomials due to Dougall [23]. However, the derivation of noncentral trivariate distribution for an arbitrary covariance matrix seems intractable with the millers approach.

This paper is organized as follows. Section II derives the generalized Rician distribution and trivariate noncentral chisquared distribution. Some simplifications related to previously known results are also discussed there. Section III deals with the derivation of cfd and chf with some simplifications. Section IV concludes the paper.

\section{Derivation of Trivariate Noncentral $\chi^{2}$ DISTRIBUTION}

Let $\left\{\mathbf{X}_{1}, \mathbf{X}_{2}, \mathbf{X}_{3}\right\}$ be three nonzero mean Gaussian vectors with $E\left(\mathbf{X}_{i}=\mathbf{a}\right)$ and $\mathbf{X}_{i}=\left(x_{1 i} x_{2 i} \ldots \ldots x_{n i}\right)^{T}$ for all $1 \leq i \leq 3$. Here $\mathbf{a}=\left(a_{1} a_{2} \ldots a_{n}\right)^{T}, E(\cdot)$ represents the mathematical expectation and $(\cdot)^{T}$ denotes the transpose of a matrix. Let $\mathbf{V}_{j}=\left(x_{j 1} x_{j 2} x_{j 3}\right)^{T}, 1 \leq j \leq n$ be independent four dimensional nonzero mean Gaussian vectors composed of the $j$ th components of $\mathbf{X}_{i}$.

In this display, the columns are the $n$-dimensional Gaussian vectors

\begin{tabular}{c|ccc} 
& $\mathbf{X}_{1}$ & $\mathbf{X}_{2}$ & $\mathbf{X}_{3}$ \\
\hline $\mathbf{V}_{1}$ & $x_{11}$ & $x_{12}$ & $x_{13}$ \\
$\mathbf{V}_{2}$ & $x_{21}$ & $x_{22}$ & $x_{23}$ \\
& $\cdots$ & $\ldots$ & $\cdots$ \\
$\mathbf{V}_{n}$ & $x_{n, 1}$ & $x_{n, 2}$ & $x_{n, 3}$
\end{tabular}


and the rows $\mathbf{V}_{j}$ are independent from each other and with identical covariance matrix $\mathbf{M}_{3}$. The inverse covariance matrix of $\mathbf{V}_{j}$ is

$$
\mathbf{W}_{3}=\mathbf{M}_{3}^{-1}=\left(\begin{array}{lll}
w_{11} & w_{12} & w_{13} \\
w_{12} & w_{22} & w_{23} \\
w_{13} & w_{23} & w_{33}
\end{array}\right)
$$

The derivation of the joint density function is analytically tractable with the Miller's approach if one or more off diagonal elements of $\mathbf{W}_{3}$ are zero. The most general such realization is the tridiagonal form of matrix or in other words $w_{13}=0$. It is well known that the exponential type covariance matrix gives a tridiagonal form of inverse matrix [24]. This assumption is common for all the multivariate derivations given in [15], [17], [18].

The amplitudes $s_{i}=\left|\mathbf{X}_{i}\right|(1 \leq i \leq 3)$, being the square root of sum of squares of $n$ nonzero mean independent Gaussian random variables, are generalized Rician random variables. Here $|\cdot|$ denotes the norm of a column vector. The joint pdf of $\left\{\mathbf{X}_{1}, \mathbf{X}_{2}, \mathbf{X}_{3}\right\}$ is clearly given by

$$
\begin{aligned}
& f\left(\mathbf{X}_{1}, \mathbf{X}_{2}, \mathbf{X}_{3}\right)=\prod_{j=1}^{n} f\left(\mathbf{V}_{\mathbf{j}}\right)=\frac{W_{3}^{\frac{n}{2}}}{(2 \pi)^{\frac{3 n}{2}}} \\
& \quad \times \exp \left\{-\frac{1}{2} \sum_{j=1}^{n}\left(\mathbf{V}_{\mathbf{j}}-a_{j} \mathbf{1}_{3}\right) \mathbf{W}_{3}\left(\mathbf{V}_{\mathbf{j}}-a_{j} \mathbf{1}_{3}\right)^{T}\right\}
\end{aligned}
$$

where $\mathbf{1}_{3}=\left(\begin{array}{lll}1 & 1 & 1\end{array}\right)$.

Expanding the quadratic form in (3) and interchanging $\mathbf{V}_{j}$ 's by $\mathbf{X}_{i}$ (see the display in (1)), we find that

$$
\begin{array}{r}
f\left(\mathbf{X}_{1}, \mathbf{X}_{2}, \mathbf{X}_{3}\right)=\frac{W_{3}^{\frac{n}{2}}}{(2 \pi)^{\frac{3 n}{2}}} \exp \left\{-\frac{1}{2}\left(w_{11} s_{1}^{2}+w_{22} s_{2}^{2}\right.\right. \\
\left.\left.+w_{33} s_{3}^{2}+w a^{2}\right)\right\} \exp \left\{\mathbf{X}_{1}^{T}\left(w_{1} \mathbf{a}-w_{12} \mathbf{X}_{2}\right)\right\} \\
\exp \left\{\mathbf{X}_{3}^{T}\left(w_{3} \mathbf{a}-w_{23} \mathbf{X}_{2}\right)\right\} \exp \left(w_{2} \mathbf{X}_{2}^{T} \mathbf{a}\right)
\end{array}
$$

where $a^{2}=|\mathbf{a}|^{2}, w_{1}=w_{11}+w_{12}, w_{2}=w_{12}+w_{23}+$ $w_{22}, w_{3}=w_{23}+w_{33}$ and $w=w_{1}+w_{2}+w_{3}$. From this pdf (4), we need to integrate out $\mathbf{X}_{i}, 1 \leq i \leq 3$, subject to the constraints $s_{i}=\left|\mathbf{X}_{i}\right|$, which will yield the joint pdf of correlated generalized Rician variables $\left\{s_{1}, s_{2}, s_{3}\right\}$ [16]. Now, the joint density can be written as

$$
\begin{gathered}
f\left(\mathbf{X}_{1}, \mathbf{X}_{2}, \mathbf{X}_{3}\right)=\frac{W_{3}^{\frac{n}{2}}}{(2 \pi)^{\frac{3 n}{2}}} \exp \left\{-\frac{1}{2}\left(w_{11} s_{1}^{2}+w_{22} s_{2}^{2}\right.\right. \\
\left.\left.+w_{33} s_{3}^{2}+w a^{2}\right)\right\} \int_{\left|\mathbf{X}_{1}\right|=s_{1}} \exp \left\{\mathbf{X}_{1}^{T}\left(w_{1} \mathbf{a}-w_{12} \mathbf{X}_{2}\right)\right\} d \sigma_{x_{1}} \\
\int_{\left|\mathbf{X}_{3}\right|=s_{3}} \exp \left\{\mathbf{X}_{3}^{T}\left(w_{3} \mathbf{a}-w_{23} \mathbf{X}_{2}\right)\right\} d \sigma_{x_{3}} \\
\times \int_{\left|\mathbf{X}_{2}\right|=s_{2}} \exp \left(w_{2} \mathbf{X}_{2}^{T} \mathbf{a}\right) d \sigma_{x_{2}}
\end{gathered}
$$

where $d \sigma_{x_{i}}, 1 \leq i \leq 3$ are the elements of surface area and $W_{3}$ denotes the determinant of the square matrix $\mathbf{W}_{3}$. The first integral in (5) can be evaluated as [16, eq.2.2.9]

$$
\begin{aligned}
\int_{\left|\mathbf{X}_{1}\right|=} & \exp \left\{\mathbf{X}_{1}^{T}\left(w_{1} \mathbf{a}-w_{12} \mathbf{X}_{2}\right)\right\} d \sigma_{x_{1}}=\left(2 \pi s_{1}\right)^{\frac{n}{2}} \\
& \times\left|w_{1} \mathbf{a}-w_{12} \mathbf{X}_{2}\right|^{\frac{2-n}{2}} I_{\frac{n-2}{2}}\left(s_{1} \mid w_{1} \mathbf{a}-w_{12} \mathbf{X}_{2}\right)
\end{aligned}
$$

where $I_{n}$ is the $n$th order modified Bessel function of the first kind [25], and the second integral follows the same form. Furthermore, the right side of (6) can be written using the generalized Neumann addition formula [25] when $n>2$ as

$$
\begin{gathered}
\left(2 \pi s_{1}\right)^{\frac{n}{2}}\left|w_{1} \mathbf{a}-w_{12} \mathbf{X}_{2}\right|^{\frac{2-n}{2}} I_{\frac{n-2}{2}}\left(s_{1} \mid w_{1} \mathbf{a}-w_{12} \mathbf{X}_{2}\right)= \\
\quad \frac{\left(2 \pi s_{1}\right)^{\frac{n}{2}} 2^{\frac{n-2}{2}} \Gamma\left(\frac{n-2}{2}\right) s_{1}}{\left(w_{1} w_{12} a s_{2}\right)^{\frac{n-2}{2}}} \sum_{k=0}^{\infty}(-1)^{k}\left(\frac{n}{2}+k-1\right) \\
\times I_{\frac{n}{2}+k-1}\left(a w_{1} s_{1}\right) I_{\frac{n}{2}+k-1}\left(w_{12} s_{1} s_{2}\right) C_{k}^{\frac{n-2}{2}}(\cos \theta)
\end{gathered}
$$

where $\Gamma(x)$ is the Gamma function [26], $C_{n}^{\lambda}(x)$ denotes the ultraspherical polynomials [26] and $\theta$ is the angle between the vectors a and $\mathbf{X}_{2}$. Following (7) we can write (5) as given in (8). The product of two ultraspherical polynomials can be written using the Dougall's identity given in [23, eq.6.8.4] as

$$
C_{p}^{\lambda}(x) C_{q}^{\lambda}(x)=\sum_{m=0}^{\min (p, q)} A(m, p, q) C_{p+q-2 n}^{\lambda}(x)
$$

$$
\begin{gathered}
f\left(s_{1}, s_{2}, s_{3}\right)=\frac{W_{3}^{\frac{n}{2}} 2^{n-2} \Gamma^{2}\left(\frac{n-2}{2}\right) s_{1} s_{3}}{(2 \pi)^{\frac{n}{2}}\left(w_{1} w_{12} w_{23} w_{3}\right)^{\frac{n-2}{2}}\left(a s_{2}\right)^{n-2}} \exp \left\{-\frac{1}{2}\left(w_{11} s_{1}^{2}+w_{22} s_{2}^{2}+w_{33} s_{3}^{2}+w a^{2}\right)\right\} \\
\times \sum_{k=0}^{\infty} \sum_{l=0}^{\infty}(-1)^{k+l}\left(\frac{n}{2}+k-1\right)\left(\frac{n}{2}+l-1\right) I_{\frac{n}{2}+k-1}\left(a w_{1} s_{1}\right) I_{\frac{n}{2}+k-1}\left(w_{12} s_{1} s_{2}\right) I_{\frac{n}{2}+l-1}\left(a w_{3} s_{3}\right) I_{\frac{n}{2}+l-1}\left(a w_{23} s_{2} s_{3}\right) \\
\int_{\left|\mathbf{X}_{2}\right|=s_{2}} \exp \left(w_{2} \mathbf{X}_{2}^{T} \mathbf{a}\right) C_{k}^{\frac{n-2}{2}}(\cos \theta) C_{l}^{\frac{n-2}{2}}(\cos \theta) d \sigma_{x_{2}}
\end{gathered}
$$

$$
A(m, p, q)=\frac{(p+q+\lambda-2 m)(\lambda)_{m}(\lambda)_{p-m}(\lambda)_{q-m}(2 \lambda)_{p+q-m}(p+q-2 m) !}{(p+q+\lambda-m) m !(p-m) !(q-m) !(\lambda)_{p+q-m}(2 \lambda)_{p+q-2 m}}
$$


where $A(m, p, q)$ is given by (10) with $(\lambda)_{n}=\frac{\Gamma(\lambda+n)}{\Gamma(\lambda)}$ denotes the Pochhammer symbol [27] and $\min (p, q)$ selects the minimum of $p, q$. We make use of the Dougall's identity in (8) to yield (11) given at the bottom of the page. The integral in (11) can be solved using [16, eq.2.2.26] to give

$$
\begin{aligned}
& \int_{\left|\mathbf{X}_{2}\right|=s_{2}} \exp \left(w_{2} \mathbf{X}_{2}^{T} \mathbf{a}\right) C_{k+l-2 m}^{\frac{n-2}{2}}(\cos \theta) d \sigma_{x_{2}}= \\
& \frac{(2 \pi)^{\frac{n}{2}} s_{2}^{n-1}\left(\begin{array}{c}
n+k+l-2 m-3 \\
n-3
\end{array}\right)}{\left(a w_{2} s_{2}\right)^{\frac{n-2}{2}}} I_{\frac{n}{2}+k+l-2 m-1}\left(a w_{2} s_{2}\right)
\end{aligned}
$$

where $\left(\begin{array}{l}n \\ r\end{array}\right)=\frac{n !}{r !(n-r) !}$. Substituting (12) in (11) and after some algebraic manipulations the joint density of trivariate generalized Rician density for $n>2$ can be written as given in (13). The case when $n=2$ is given in [22, eq.3].

Since we are interested in the trivariate noncentral $\chi^{2}$ distribution, the following variable transformations are introduced in (13), $r_{1}=s_{1}^{2}, r_{2}=s_{2}^{2}, r_{3}=s_{3}^{2}$. Now it is clear that $\left\{r_{1}, r_{2}, r_{3}\right\}$ represent the noncentral $\chi^{2}$ variables. After some algebraic manipulation the trivariate non-central $\chi^{2}$ distribution can be written as given in (14) at the bottom of the page. To the best of the authors' knowledge (14) is a novel result. Even though (14) is not valid for $n=2$ the degenerated cases of (14) valid for all $n \geq 2$ as shown below. Moreover, if a given covariance matrix does not match with the criteria mentioned above, we can use the Green's matrix approach given in [18] to approximate the given covariance matrix with a matrix having tridiagonal form of inverse. Next some simplifications of (14) are given.

\section{A. Independent noncentral $\chi^{2}$ distributions}

It is obvious that $\mathbf{W}_{3}$ is a diagonal matrix with the elements $\left\{w_{11}, w_{22}, w_{33}\right\}$ under this scenario. Since all off diagonal elements are zero, we can obtain the following two important limits involving the Bessel functions

$$
\begin{aligned}
\lim _{w_{12} \rightarrow 0} \frac{I_{\frac{n}{2}+k-1}\left(w_{12} \sqrt{r_{1} r_{2}}\right)}{w_{12}^{\frac{n-2}{2}}} & =\frac{\left(\frac{\sqrt{r_{1} r_{2}}}{2}\right)^{\frac{n}{2}-1}}{\Gamma\left(\frac{n}{2}\right)} \\
\lim _{w_{23} \rightarrow 0} \frac{I_{\frac{n}{2}+l-1}\left(w_{23} \sqrt{r_{2} r_{3}}\right)}{w_{23}^{\frac{n-2}{2}}} & =\frac{\left(\frac{\sqrt{r_{2} r_{3}}}{2}\right)^{\frac{n}{2}-1}}{\Gamma\left(\frac{n}{2}\right)}
\end{aligned}
$$

which are valid if $k=0, l=0$. Substituting (15), (16) in (14) and after some rearrangements with little algebra we get

$$
\begin{aligned}
& g\left(r_{1}, r_{2}, r_{3}\right)=\prod_{i=1}^{3} \frac{w_{i i}}{2}\left(\frac{r_{i}}{a^{2}}\right)^{\frac{n-2}{4}} \exp \left\{-\frac{w_{i i}}{2}\left(r_{i}+a^{2}\right)\right\} \\
& \times I_{\frac{n}{2}-1}\left(a w_{i i} \sqrt{r_{i}}\right) .
\end{aligned}
$$

extension for central $\chi^{2}$ distribution follows from (17).

$$
\begin{aligned}
& f\left(s_{1}, s_{2}, s_{3}\right)=\frac{W_{3}^{\frac{n}{2}} 2^{n-2} \Gamma^{2}\left(\frac{n-2}{2}\right) s_{1} s_{3}}{(2 \pi)^{\frac{n}{2}}\left(w_{1} w_{12} w_{23} w_{3}\right)^{\frac{n-2}{2}}\left(a s_{2}\right)^{n-2}} \exp \left\{-\frac{1}{2}\left(w_{11} s_{1}^{2}+w_{22} s_{2}^{2}+w_{33} s_{3}^{2}+w a^{2}\right)\right\} \\
& \times \sum_{k=0}^{\infty} \sum_{l=0}^{\infty} \sum_{m=0}^{\min (k, l)}(-1)^{k+l}\left(\frac{n}{2}+k-1\right)\left(\frac{n}{2}+l-1\right) A(m, k, l) I_{\frac{n}{2}+k-1}\left(a w_{1} s_{1}\right) I_{\frac{n}{2}+k-1}\left(w_{12} s_{1} s_{2}\right) I_{\frac{n}{2}+l-1}\left(a w_{3} s_{3}\right) \\
& \quad \times I_{\frac{n}{2}+l-1}\left(a w_{23} s_{2} s_{3}\right) \int_{\left|\mathbf{X}_{2}\right|=s_{2}} \exp \left(w_{2} \mathbf{X}_{2}^{T} \mathbf{a}\right) C_{k+l-2 m}^{\frac{n-2}{2}}(\cos \theta) d \sigma_{x_{2}}
\end{aligned}
$$

$$
\begin{aligned}
& f\left(s_{1}, s_{2}, s_{3}\right)=\frac{W_{3}^{\frac{n}{2}} 2^{n-2} \Gamma^{2}\left(\frac{n-2}{2}\right) s_{1} s_{2} s_{3}}{\left(w_{1} w_{2} w_{3} w_{12} w_{23} a s_{2}\right)^{\frac{n-2}{2}}(a)^{n-2}} \exp \left\{-\frac{1}{2}\left(w_{11} s_{1}^{2}+w_{22} s_{2}^{2}+w_{33} s_{3}^{2}+w a^{2}\right)\right\} \\
& \times \sum_{k=0}^{\infty} \sum_{l=0}^{\infty} \sum_{m=0}^{\min (k, l)}(-1)^{k+l}\left(\begin{array}{c}
n+k+l-2 m-3 \\
n-3
\end{array}\right) A(m, k, l)\left(\frac{n}{2}+k-1\right)\left(\frac{n}{2}+l-1\right) I_{\frac{n}{2}+k-1}\left(a w_{1} s_{1}\right) \\
& \times I_{\frac{n}{2}+k-1}\left(w_{12} s_{1} s_{2}\right) I_{\frac{n}{2}+l-1}\left(a w_{3} s_{3}\right) I_{\frac{n}{2}+l-1}\left(a w_{23} s_{2} s_{3}\right) I_{\frac{n}{2}+k+l-2 m-1}\left(a w_{2} s_{2}\right) \\
& g\left(r_{1}, r_{2}, r_{3}\right)=\frac{W_{3}^{\frac{n}{2}} 2^{n-5} \Gamma^{2}\left(\frac{n-2}{2}\right)}{\left(w_{1} w_{2} w_{3} w_{12} w_{23}\right)^{\frac{n-2}{2}}(a)^{\frac{3 n}{2}-3}\left(\sqrt{r_{2}}\right)^{\frac{n-2}{2}}} \exp \left\{-\frac{1}{2}\left(w_{11} s_{1}^{2}+w_{22} s_{2}^{2}+w_{33} s_{3}^{2}+w a^{2}\right)\right\} \\
& \times \sum_{k=0}^{\infty} \sum_{l=0}^{\infty} \sum_{m=0}^{m i n(k, l)}(-1)^{k+l}\left(\begin{array}{c}
n+k+l-2 m-3 \\
n-3
\end{array}\right) A(m, k, l)\left(\frac{n}{2}+k-1\right)\left(\frac{n}{2}+l-1\right) I_{\frac{n}{2}+k-1}\left(a w_{1} \sqrt{r_{1}}\right) \\
& \times I_{\frac{n}{2}+k-1}\left(w_{12} \sqrt{r_{1} r_{2}}\right) I_{\frac{n}{2}+l-1}\left(a w_{3} \sqrt{r_{3}}\right) I_{\frac{n}{2}+l-1}\left(a w_{23} \sqrt{r_{2} r_{3}}\right) I_{\frac{n}{2}+k+l-2 m-1}\left(a w_{2} \sqrt{r_{2}}\right)
\end{aligned}
$$




\section{B. Bivariate noncentral $\chi^{2}$ distribution}

If $\left\{r_{1}, r_{2}\right\}$ are independent from $r_{3}$, then we can write the trivariate density as a product of bivariate and univariate densities. Equating $w_{23}$ to zero and using the limit (15) with $l=0$ followed by some manipulations we get the bivariate density as given in (18). Here $W_{2}$ denotes the determinant of $2 \times 2$ inverse covariance matrix $\mathbf{W}_{2}$. Equation (18) is equivalent to the previously published result by Miller [16, eq.2.2.18].

\section{CDF AND CHF of TRIVARIATE DENSITY}

\section{A. Cumulative Distribution Function}

The trivariate cdf is by definition [28]

$$
G\left(r_{1}, r_{2}, r_{3}\right)=\int_{0}^{r_{1}} \int_{0}^{r_{2}} \int_{0}^{r_{3}} g\left(y_{1}, y_{2}, y_{3}\right) d y_{1} d y_{2} d y_{3} .
$$

Substituting (14) in (19), followed by expansion of the Bessel function term with its infinite series representation and subsequent term by term integration, we get the cdf of trivariate noncentral $\chi^{2}$ distribution as given in (20) with $\lambda_{1}=2 i_{1}+k$, $\lambda_{2}=2 i_{5}+k+l-2 m, \lambda_{3}=2 i_{3}+l, \lambda_{4}=2 i_{2}+k, \lambda_{5}=2 i_{4}+l$, $\delta_{1}=i_{1}+i_{2}+k+\frac{n}{2}, \delta_{2}=i_{2}+i_{4}+i_{5}+k+\frac{n}{2}-m$, $\delta_{3}=i_{3}+i_{4}+l+\frac{n}{2}$ and $\gamma(a, x)=\int_{0}^{x} t^{a-1} \exp (-t) d t$ is the incomplete gamma function [27]. Moreover if $n>2$ is an even integer then we would have used an alternative closed form expression given in [19] instead of incomplete gamma function. Simplification for special cases are straightforward with (20).

\section{B. Characteristic Function}

The joint chf is defined as [28]

$$
\psi\left(v_{1}, v_{2}, v_{3}\right)=E\left\{\exp \left(v_{1} r_{1} j+v_{2} r_{2} j+v_{3} r_{3} j\right)\right\}
$$

where $j=\sqrt{-1}$. Following the same line of arguments as for the cdf derivation, we encounter integrals of the form

$$
\int_{0}^{\infty} x^{\nu-1} \exp (-[p+j q] x) d x
$$

which can be solved using [26, eq.3.381.5] to yield the chf as given in (22). The bivariate generalization is straightforward with (22). If all $\left\{r_{1}, r_{2}, r_{3}\right\}$ are independent then the joint chf can be written as a product of individual chfs. The chf of univariate $\chi^{2}$ distribution has a well known closed form [19, eq.2.1.117]. We end up with a product of three infinite series of the form

$$
\begin{array}{r}
\psi\left(v_{1}\right)=\sum_{i_{1}=0}^{\infty} \frac{w_{11}^{2 i_{1}+\frac{n}{2}} a^{2 i_{1}}}{2^{2 i_{1}+\frac{n}{2}} i_{1} !\left(\frac{w_{11}^{2}}{4}+v_{1}^{2}\right)^{i_{1}+\frac{n}{2}}} \\
\times e^{\left\{j\left(i_{1}+\frac{n}{2}\right) \arctan \left(\frac{2 v_{1}}{w_{11}}\right)\right\}}
\end{array}
$$

$$
\begin{array}{r}
g\left(r_{1}, r_{2}\right)=\frac{W_{2}^{\frac{n}{2}} 2^{\frac{n}{2}-3} \Gamma\left(\frac{n-2}{2}\right)}{\left(w_{1} w_{2} w_{12}\right)^{\frac{n-2}{2}}(a)^{n-2}} \exp \left\{-\frac{1}{2}\left(w_{11} s_{1}^{2}+w_{22} s_{2}^{2}+\left[w_{1}+w_{2}\right] a^{2}\right)\right\} \sum_{k=0}^{\infty}(-1)^{k}\left(\begin{array}{c}
n+k-3 \\
n-3
\end{array}\right)\left(\frac{n}{2}+k-1\right) \\
\times I_{\frac{n}{2}+k-1}\left(a w_{1} \sqrt{r_{1}}\right) I_{\frac{n}{2}+k-1}\left(w_{12} \sqrt{r_{1} r_{2}}\right) I_{\frac{n}{2}+k-1}\left(a w_{2} \sqrt{r_{2}}\right)
\end{array}
$$

$$
\begin{gathered}
G\left(r_{1}, r_{2}, r_{3}\right)=W_{3}^{\frac{n}{2}} \Gamma^{2}\left(\frac{n-2}{2}\right) \exp \left(-\frac{a^{2} w}{2}\right) \sum_{k, l=0}^{\infty} \sum_{m=0}^{\min (k, l)} \sum_{i_{1}, i_{2}, i_{3}, i_{4}, i_{5}=0}^{\infty}(-1)^{k+l} \frac{A(m, k, l)\left(\begin{array}{c}
n+k+l-2 m-3 \\
n-3
\end{array}\right)}{2^{\frac{1}{2}\left(\lambda_{1}+\lambda_{2}+\lambda_{3}\right)} i_{1} ! i_{2} ! i_{3} ! i_{4} ! i_{5} !} \\
\times \frac{\left(\frac{n}{2}+k-1\right)\left(\frac{n}{2}+l-1\right) a^{\lambda_{1}+\lambda_{2}+\lambda_{3}} w_{1}^{\lambda_{1}} w_{2}^{\lambda_{2}} w_{3}^{\lambda_{3}} w_{12}^{\lambda_{4}} w_{23}^{\lambda_{5}}}{\Gamma\left(i_{1}+\frac{n}{2}+k\right) \Gamma\left(i_{2}+\frac{n}{2}+k\right) \Gamma\left(i_{3}+\frac{n}{2}+l\right) \Gamma\left(i_{4}+\frac{n}{2}+1\right) \Gamma\left(i_{5}+\frac{n}{2}+k+l-2 m\right) w_{11}^{\delta_{1}} w_{22}^{\delta_{2}} w_{33}^{\delta_{3}} \gamma\left(\delta_{1}, \frac{w_{11}}{2} r_{1}\right)} \\
\times \gamma\left(\delta_{2}, \frac{w_{22}}{2} r_{2}\right) \gamma\left(\delta_{3}, \frac{w_{33}}{2} r_{3}\right)
\end{gathered}
$$

$$
\begin{aligned}
& \psi\left(v_{1}, v_{2}, v_{3}\right)=W_{3}^{\frac{n}{2}} \Gamma^{2}\left(\frac{n-2}{2}\right) \exp \left(-\frac{a^{2} w}{2}\right) \sum_{k, l=0}^{\infty} \sum_{m=0}^{\min (k, l)} \sum_{i_{1}, i_{2}, i_{3}, i_{4}, i_{5}=0}^{\infty}(-1)^{k+l} \frac{A(m, k, l)\left({ }^{n+k+l-2 m-3}\right)}{2^{\left(\lambda_{1}+\lambda_{2}+\lambda_{3} \lambda_{4}+\lambda_{5}\right)-\frac{3 n}{2}} i_{1} ! i_{2} ! i_{3} ! i_{4} ! i_{5} !} \\
& \left(\frac{n}{2}+k-1\right)\left(\frac{n}{2}+l-1\right) a^{\lambda_{1}+\lambda_{2}+\lambda_{3}} w_{1}^{\lambda_{1}} w_{2}^{\lambda_{2}} w_{3}^{\lambda_{3}} w_{12}^{\lambda_{4}} w_{23}^{\lambda_{5}} \Gamma\left(\delta_{1}\right) \Gamma\left(\delta_{2}\right) \Gamma\left(\delta_{3}\right) \\
& \Gamma\left(i_{1}+\frac{n}{2}+k\right) \Gamma\left(i_{2}+\frac{n}{2}+k\right) \Gamma\left(i_{3}+\frac{n}{2}+l\right) \Gamma\left(i_{4}+\frac{n}{2}+1\right) \Gamma\left(i_{5}+\frac{n}{2}+k+l-2 m\right)\left(\frac{w_{11}^{2}}{4}+v_{1}^{2}\right)^{\frac{\delta_{1}}{2}} \\
& \times \frac{1}{\left(\frac{w_{22}^{2}}{4}+v_{2}^{2}\right)^{\frac{\delta_{2}}{2}}\left(\frac{w_{33}^{2}}{4}+v_{3}^{2}\right)^{\frac{\delta_{3}}{2}}} \exp \left\{j \delta_{1} \arctan \left(\frac{2 v_{1}}{w_{11}}\right)+j \delta_{2} \arctan \left(\frac{2 v_{2}}{w_{22}}\right)+j \delta_{3} \arctan \left(\frac{2 v_{3}}{w_{33}}\right)\right\}
\end{aligned}
$$




$$
\begin{gathered}
\psi\left(v_{2}\right)=\sum_{i_{5}=0}^{\infty} \frac{w_{22}^{2 i_{5}+\frac{n}{2}} a^{2 i_{5}}}{2^{2 i_{5}+\frac{n}{2}} i_{5} !\left(\frac{w_{22}^{2}}{4}+v_{2}^{2}\right)^{i_{5}+\frac{n}{2}}} \\
\times e^{\left\{j\left(i_{5}+\frac{n}{2}\right) \arctan \left(\frac{2 v_{2}}{w_{22}}\right)\right\}} \\
\psi\left(v_{3}\right)=\sum_{i_{3}=0}^{\infty} \frac{w_{33}^{2 i_{3}+\frac{n}{2}} a^{2 i_{3}}}{2^{2 i_{3}+\frac{n}{2}} i_{3} !\left(\frac{w_{33}^{2}}{4}+v_{3}^{2}\right)^{i_{3}+\frac{n}{2}}} \\
\times e^{\left\{j\left(i_{3}+\frac{n}{2}\right) \arctan \left(\frac{2 v_{3}}{w_{33}}\right)\right\}}
\end{gathered}
$$

if we assume $w_{12}=w_{23}=0$ (i.e., $i_{2}=i_{4}=k=l=0$ ) in (22) and the closed form solution for chf is not immediately obvious. Nevertheless, it is easy to see that the individual infinite summations are in the form of exponential series and by using little algebra one can show that the individual chf series reduces to [19, eq.2.1.117].

\section{CONCLUSION}

A new infinite series representation for the trivariate noncentral $\chi^{2}$ distribution has been derived when the underlying Gaussian components have tridiagonal form of inverse covariance matrix. An identity for a product of two ultrspherical polynomials due to Dougall and Miller's approach are used in the derivation. Moreover, the chf and cdf series are also derived. Some special case of the joint density function are also discussed. However, the derivation for an arbitrary covariance matrix seems intractable with this approach.

\section{ACKNOWLEDGMENT}

The first author would like to thank the government of Finland for the doctoral scholarship provided to him.

\section{REFERENCES}

[1] M. K. Simon and M. -S. Alouini, "On the difference of two chisquared variates with application to outage probability computation," IEEE Trans. Commun., vol. 49, no. 11, pp. 1946-1954, Nov. 2001.

[2] G. J. Foschini and Gans M, "On the limit of wireless communication in a fading environment when using multiple antennas," Wireless Pers. Commun., vol. 6, pp. 311-335, Mar. 1998.

[3] D. A. Gore, R. W. Heath, and A. J. Paulraj, "Transmit selection in spatial multiplexing systems," IEEE Commun. Lett., vol. 6, no. 11, pp. 491-493, Nov. 2002.

[4] M. K. Simon and M. -S. Alouini, Digital Communication over Fading Channels, New Jersey: John Wiley \& Sons, Inc. 2005.

[5] H. Jafarkhani, Space-Time Coding, Cambridge University Press. 2005.
[6] T. Cacoullos and M. Koutras, "Quadratic forms in spherical random variables: Generalized non-central $\chi^{2}$ distribution," Naval Res. Logist. Quart., vol. 31, pp. 447-461, 1984.

[7] M. Koutras, "On the generalized noncentral chi-squared distribution induced by elliptical gamma law,” Biometrika, vol. 73, pp. 528-532, 1986.

[8] P. B. Patnaik, "The non-central $\chi^{2}$ and F-distributions and their applications," Biometrika, vol. 36, pp. 202-232, 1949.

[9] M. L. Tiku, "Laguerre series forms of non-central $\chi^{2}$ and Fdistributions," Biometrika, vol. 52, pp. 415-427, 1965.

[10] K. H. Biyari and W. C. Lindsey, "Statistical distributions of Hermitian quadratic forms in complex Gaussian variables," IEEE Trans. Inform. Theory, vol. 39, no. 3, pp. 1076-1082, May. 1993.

[11] M. Hagedorn, P. J. Smith, P. J. Bones, R. P. Millane, and D. Pairman, "A trivariate chi-squared distribution derived from the complex Wishart distribution," J. Multivariate Anal., vol. 97, pp. 655-674, 2006.

[12] D. R. Jensen, "The joint distribution of traces of Wishart matrices and some applications," Ann. Math. Stat., vol. 41, pp. 133-145, 1970.

[13] P. R. Krishnaiah, P. Hagis, and L. Steinberg, "A note on the bivariate chi distribution," SIAM Rev., vol. 5, pp. 140-144, 1963.

[14] T. Royen, "Expansions for the multivariate chi-squared distribution," $J$. Multivariate Anal., vol. 38, pp. 213-232, 1991.

[15] L. E. Blumenson and K. S. Miller, "Properties of generalized Rayleigh distributions," Ann. Math. Statis., vol. 34, pp. 903-910, 1963.

[16] K. S. Miller, Multidimensional Gaussian Distributions, John Wiley, 1964.

[17] G. K. Karagiannidis, D. A. Zogas, and S. A. Kotsopoulos, "On the multivariate Nakagami- $m$ distribution with exponential correlation," IEEE Trans. Commun., vol. 51, pp. 1240-1244, Aug. 2003.

[18] G. K. Karagiannidis, D. A. Zogas, and S. A. Kotsopoulos, "An efficient approach to multivariate Nakagami- $m$ distribution using Green's matrix approximation," IEEE Trans. Wireless Commun., vol. 2, pp. 883-889, Sep. 2003.

[19] J. G. Proakis, Digital Communications, 3rd ed., McGraw-Hill, 1995.

[20] D. Middleton, An Introduction to Statistical Communication Theory. New York: McGraw-Hill, 1960.

[21] M. K. Simon, "Comments on "Infinite-series representations associated with the bivariate Rician distribution and their applications"," IEEE Trans. Commun., vol. 54, no. 8, pp. 1511-1512, Aug. 2006.

[22] K. D. P. Dharmawansa, R. M. A. P. Rajatheva, and K. M. Ahmed, "On the bivariate and trivariate Rician distributions," in Proc. IEEE VTC 2006, Sept. 25-28.

[23] G. E. Andrews, R. Askey, and R. Roy, Special Functions, vol. 71, Cambridge University Press, 1999.

[24] V. A. Aalo, "Performance of maximal-ratio diversity systems in a correlated Nakagami-fading environment," IEEE Trans. Commun., vol. 43 , pp. 2360-2369, Aug. 1995.

[25] G. N. Watson, A Treatise on the Theory of Bessel Functions, Cambridge, U. K.: Cambridge Univ. Pres, 1944.

[26] I. S. Gradshteyn and I. M. Ryzhik, Tables of Integrals, Series and Products, 4th ed. New York: Academic Press, 1980.

[27] L. C. Andrews, Special Function of Mathematics for Engineers, 2nd ed. Bellignham: SPIE Press, 1998.

[28] A. Papoulis and S. U. Pillai, Probability, Random Variables and Stochastic Processes, 4th ed. New York: McGraw-Hill, 2002. 